A.J. Morales, J. Reyes, K.A. Ahmed, I. Boxx, The Effects of Turbulence on the Lean Blowout Mechanisms of Bluff-Body Flames, Proc. Combust. Inst. 38 (2021) 6317-6325.

The original publication is available at www.sciencedirect.com

https://dx.doi.org/10.1016/j.proci.2020.06.138

(C) <2021>. This manuscript version is made available under the CC-BY-NC-ND

4.0 license http://creativecommons.org/licenses/by-nc-nd/4.0/ 


\section{The Effects of Turbulence on the Lean Blowout Mechanisms of Bluff-Body Flames}

Anthony J. Morales ${ }^{\mathrm{a}, \mathrm{b}}$, Jonathan Reyes ${ }^{\mathrm{a}, \mathrm{c}}$, Kareem A. Ahmed ${ }^{\mathrm{a}, \mathrm{d}}$, Isaac Boxx ${ }^{\mathrm{b}, \mathrm{e}}$

${ }^{a}$ Center for Advanced Turbomachinery \& Energy Research, Department of Mechanical and Aerospace

Engineering, University of Central Florida, Orlando, Florida, 32816, USA

${ }^{\mathrm{b}}$ Institute of Combustion Technology of the German Aerospace Center, Deutsches Zentrum für Luft-und Raumfahrt (DLR), Institut für Verbrennungstechnik, Stuttgart, Germany

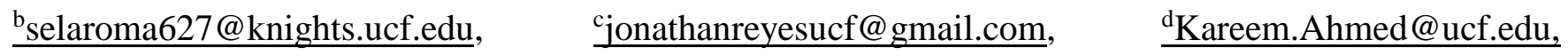

Isaac.Boxx@dlr.de

Corresponding Author: Kareem A. Ahmed

Mailing Address: 12760 Pegasus Blvd, P.O. Box 162450, Orlando, FL, 32816-2450, USA

E-mail: Kareem.Ahmed@ucf.edu

Colloquium: Gas Turbines and Rocket Engine Combustion

Alternate Colloquium: Turbulent Flames

Total Paper Length: 6191

Method of Determination: Method 1

Main Text: 3634

Equations: 46

Nomenclature: $n / a$

References: 472

Tables: 106

Figures and Captions Total: 1933

Fig 1: 201, Fig 2: 166, Fig 3: 160, Fig 4: 256, Fig 5: 285, Fig 6: 313, Fig 7: 166, Fig 8: 248, Fig 9: 138 


\section{List of Captions}

Figure 1: (a) Bluff-body combustion facility, (b) bluff-body, (c) turbulence generator, and (d) schlieren image of choked impinging jets from the turbulence generator.

Figure 2: Temporal equivalence ratio throughout the extinction duration for all turbulence test conditions. Symbols represent the equivalence ratio at blowout.

Figure 3: Combustion regime diagram for blowout test conditions.

Figure 4: Instantaneous flame boundary, vorticity field, and local flame strain rate for all turbulent test cases.

Figure 5: (a) Sketch of shear layer width and (b) mean transverse flame position and shear layer width through time (both quantities spatially averaged rom $2 \leq x / H \leq 4$ ).

Figure 6: (a) Strain rate along the flame, spatially averaged between $2 \leq \mathrm{x} / \mathrm{H} \leq 4$, throughout the extinction duration. (b) Karlovitz number along the flame throughout extinction.

Figure 7: PDF of flame curvature for all turbulent test cases.

Figure 8: Instantaneous flame boundary, vorticity field*, and flame strain rate obtained from the stereo PIV. The dashed blue line represents the boundary of the shear layer. *A portion of the freestream vorticity has been removed in some frames for legible annotations.

Figure 9: Comparison of the $\partial u / \partial y$ velocity gradient between the freestream and the shear layer.

Table 1: Test conditions for lean blowout experiments. $\operatorname{Re}_{T}=u_{r m s}^{\prime} L_{11} / S_{L} l_{f} . K a=\left(l_{f} / L_{11}\right)^{1 / 2}\left(u_{r m s}^{\prime} / S_{L}\right)^{3 / 2} . D a$ $=\left(L_{11} / u_{r m s}^{\prime}\right) /\left(l_{f} / S_{L}\right) . S_{T}$ determined from Peters correlation $[15,16]$. 


\begin{abstract}
The lean blowout mechanisms of premixed bluff-body flames are experimentally investigated at various turbulence intensities. Turbulence levels are varied using a novel turbulence generator, which combines static grid and fluidic jet impingement techniques. Three different turbulence levels are probed to study their effects on lean blowout. The three conditions span across the combustion regime diagram, from flamelets to broken reactions. For all three turbulence levels, the lean blowout process is induced through controlled fuel flow rate reduction. The transient blowout process is captured using three simultaneous high-speed diagnostic systems: particle image velocimetry (PIV), stereoscopic PIV (SPIV), and $\mathrm{C}_{2}{ }^{*} / \mathrm{CH}^{*}$ species measurements. The two PIV systems are used to resolve the instantaneous velocity and vorticity fields, and the $\mathrm{C}_{2}{ }^{*} / \mathrm{CH}^{*}$ species diagnostics allow for global equivalence ratios to be evaluated throughout the duration of blowout. The results show that the dynamics of lean blowout vary with turbulence intensity. At low turbulence levels, the flame experiences a global effect where the flame boundary interacts with the shear layer vorticity. This imparts high strain rates along the length of the flame, leading to blowout. As turbulence levels increase, the blowout mechanism becomes less dependent on flame and shear layer interactions and more driven by flame-turbulence interactions. At high turbulence conditions, flame-eddy interactions within the freestream augment flame stretching via increased flame straining and small-scale flame corrugations. Increased flame stretching disrupts the flame stabilization process, and ultimately results in blowout.
\end{abstract}

\title{
Keywords:
}

Lean Blowout, Bluff-Body Flames, Turbulent Flames, Flame Stabilization and Extinction 


\section{Introduction}

Lean combustion is desirable in gas turbines and aero-propulsion technologies to improve engine efficiency and limit emissions [1]. However, lean combustion processes are highly sensitive to flow-flame instabilities, which can lead to localized extinctions or global flame blowout [1,2]. For this reason, the continued study of flame stabilization, lean flame instabilities, and extinction mechanisms will promote more efficient and reliable combustion technologies.

Ramjets and turbojet afterburners frequently rely on bluff-bodies to stabilize flames within highvelocity streams [2]. However, there is a finite operational envelope in which bluff-body flames can be stabilized [3-5]. The operational window is dependent on several variables, including equivalence ratio, mass flow rate and freestream turbulence intensities $[5,6]$. When operating at lean conditions, fluctuations in any of these parameters can drive the flame beyond the stability limits and increase the likelihood of global blowout. For this reason, it is important to understand the dynamic processes that can drive the transition from stable operation to blowout.

Many studies have focused on characterizing the dynamics of bluff-body flames undergoing blowout. As the equivalence ratio is reduced, lean blowout occurs as a result of the flame speed and the baroclinic torque production both decreasing [7-9]. The reduced flame speed causes the flame boundary to reside closer to the shear layer regions near the bluff-body centerline. This results in an overlap between the flame boundary and the shear layer vorticity regions. The intense velocity gradients within the shear layers invokes high strain rates along the flame boundary which stretch the flame and cause localized extinctions to occur $[2,9,10]$. If the equivalence ratio continues to decrease, the flame and shear layer interaction will intensify and there will be a greater number of localized extinction events along the flame 
$[7,8,11]$. This will continue to occur until a majority of the flame is extinguished and the recirculation zone can no longer reignite-incoming reactants; this is when global blowout occurs $[2,7,8,11]$.

Additional studies have highlighted the effects of increased turbulence intensities on the lean blowout process. When analyzing the lower stability limits for bluff-body stabilized flames, Ballal et al [5] has shown that the blowout equivalence ratio increases with freestream turbulence intensities. This conclusion was expanded on by Chowdhury et al [12], who demonstrated that increasing the freestream turbulence intensity resulted in higher strain rates and curvature values along the flame. The combination of increased straining and curvature enhances flame stretching, and causes a greater portion of the flame to undergo extinction at high turbulence levels [12]. Combined, these two studies theorize that increasing turbulence levels lead to a greater likelihood for blowout to occur.

The goal of this paper is to explore the effects of increased turbulence on the spatiotemporal mechanisms of lean blowout. A bluff-body combustor and dynamic turbulence generator are coupled with high-speed PIV, stereoscopic PIV, and $\mathrm{C}_{2}{ }^{*} / \mathrm{CH}^{*}$ chemiluminescence diagnostics to capture the transient blowout process. These diagnostics allow for a detailed study of the flame-flow interactions that result in flame straining and lead to global blowout. The results show that increasing the freestream turbulence enhances the straining along the flame and ultimately expedites the blowout process.

\section{Experimental Methods}

\subsection{Experimental facility \& blowout methodology}

Lean blowout is studied using a high-speed combustion facility, as shown in Fig.1a. The combustor operates at atmospheric pressure and includes a turbulence generator, a ballistic type bluff-body (Fig. 1b), and an optical viewing section. The optical section has a rectangular cross-section with a length, height, 
and depth (into the page) of $260 \mathrm{~mm}, 45 \mathrm{~mm}$, and $127 \mathrm{~mm}$. The height of the bluff-body is $H=16 \mathrm{~mm}$, providing a blockage ratio of $35 \%$.

The combustor air flow rate is regulated with a Venturi flowmeter, pressure transducer, and PID controller system to maintain the desired velocity. Propane is premixed with the air flow, upstream of the combustor. The propane is metered with a pressure regulator, rotameter, and a control solenoid valve. The uncertainty in the propane and air flow rates are $4.96 \times 10^{-4} \mathrm{~kg} / \mathrm{s}$. For all test cases, the premixed propane-air mixture enters the combustor at an equivalence ratio of $\Phi=0.7$. Once the mixture is ignited, the lean
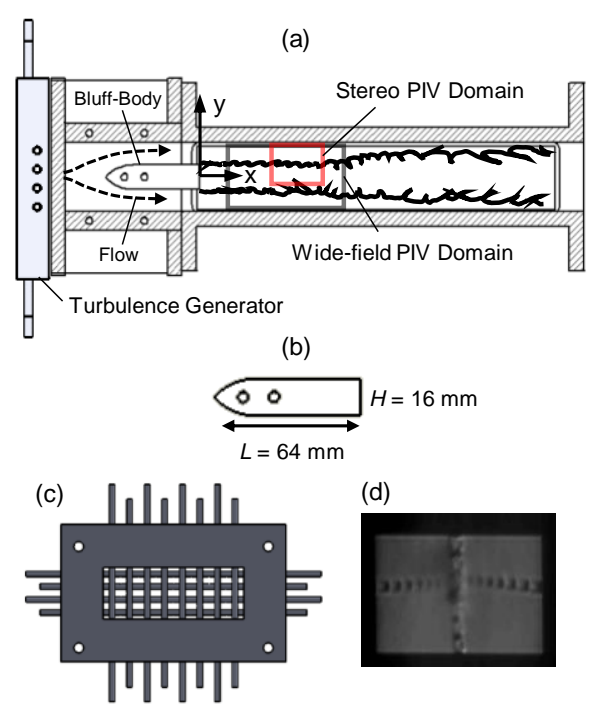

Figure 1 (a) Bluff-body combustion facility, (b) bluff-body, (c) turbulence generator, and (d) schlieren image of choked impinging jets from the turbulence generator.

flame is maintained for a short duration $(\sim 2 s)$ to mitigate any effects of preheating the bluff-body prior blowout, maintaining a bluff-body temperature of $\sim 300 \mathrm{~K}$.

Lean blowout is accomplished using the technique employed by Chaudhuri et al [8], Tuttle et [7], and Kariuki et al [13], where the fuel flow into the reactant stream is decreased to induce blowout. The fuel reduction is accomplished using a timed control system that closes the solenoid valve in the propane injection line. The time duration for the valve to close can be manually adjusted to ensure that the full blowout process occurs repeatably within the experimental duration of $250 \mathrm{~ms}$. The valve closing time is $\sim 200 \mathrm{~ms}$ which is 12-200 times larger than the chemical and flow time scales [2]. This ensures that the decrease of the fuel flow rate is adequate for capturing the relevant physics during blowout $[2,7,8,12,13]$.

\subsection{Turbulence Tailoring}


The turbulence generator shown in Fig. 1b was used to control the turbulence levels within the combustor. The design combines a static mesh grid with impinging premixed supersonic jets as shown in the schlieren image, Fig. 1c. The grid consists of 12 total tubes, each having inner and outer diameters of $4 \mathrm{~mm}$ and $4.4 \mathrm{~mm}$, and are configured as a rectangular mesh of $15.9 \mathrm{~mm} \times 11.3 \mathrm{~mm}$.

To achieve fluid jet impingement, premixed propane and air (at $\Phi=0.7$ ) are fed into both ends of each tube, and the mixture is ejected through $0.8 \mathrm{~mm}$ impingement holds drilled along the length of each tube. The impingement holes are oriented orthogonal to the bulk flow and evenly spaced in the horizontal and vertical directions. At high flow rates, the jet flow is choked generating a supersonic under-expanded premixed jet plume. The schlieren imaging is used to verify the supersonic jet structures and impingement as demonstrated in Fig. 1c. These jets interact with the premixed freestream that is flowing orthogonally (out of the page on Fig. 1b), inducing intense turbulence.

The specific turbulence conditions explored in this study are outlined in Table 1. The blowout experiments were repeated 10 times for each turbulence condition (30 trials total) to ensure that results were repeatable. For each case, the mean freestream velocity is $25 \mathrm{~m} / \mathrm{s}$. The turbulent velocity fluctuations $\left(u_{r m s}^{\prime}\right)$ and integral length scales $\left(L_{11}\right)$ are provided in Table 1 and are both evaluated within the freestream domain outside of the flame boundary. Additionally, spectral analysis has confirmed that the freestream turbulence for the Mid and High cases closely follow Kolmogrov's (-5/3) power law. The equivalence ratio at blowout $\left(\Phi_{B O}\right)$, turbulent Reynolds number $\left(R e_{T}\right)$, and Damköhler number $(D a)$ are also presented in Table 1. The $\operatorname{Re}_{T}$ and $D a$ quantities are also presented as a range of values to account for the change in flame thickness and flame speed with the decreasing equivalence ratio [14]; the initial value is for $\Phi=0.7$ and the final value is for the equivalence ratio at blowout. The turbulent flame speed $\left(S_{T}\right)$ is defined as the velocity of the flame normal to itself, and is calculated from the Peters correlation $[15,16]$; the range of $S_{T}$ 
is provided in Table 1. The last column in Table 1 documents the mean blowout duration for each test case $\left(t_{e x t}\right)$ as well as the standard deviation $(\sigma)$ among the 10 trials for each turbulence level. The total blowout duration was calculated in a similar fashion used in $[10,11,13]$, i.e. by identifying the time required for the $\mathrm{C}_{2} * / \mathrm{CH}^{*}$ chemiluminescence intensity to decrease from a maximum stable value to a minimum constant value at blowout.

Table 1. Test conditions for lean blowout experiments. $R e_{T}=u_{r m s}^{\prime} L_{11} / S_{L} l_{f} . D a=\left(L_{11} / u_{r m s}^{\prime}\right) /\left(l_{f} / S_{L}\right) . S_{T}$ determined from Peters correlation $[15,16]$.

\begin{tabular}{|c|c|c|c|c|c|c|c|c|}
\hline Test Case & $\begin{array}{c}\text { Turbulence } \\
\text { generator }\end{array}$ & $\begin{array}{c}u_{r m s}^{\prime} \\
(\mathrm{m} / \mathrm{s})\end{array}$ & $\begin{array}{c}L_{11} \\
\left(\mathrm{~m} \cdot 10^{3}\right)\end{array}$ & $\Phi_{B O}$ & $\operatorname{Re}_{T}$ & $S_{T}(\mathrm{~m} / \mathrm{s})$ & $D a$ & $\begin{array}{c}t_{\text {ext }}(\mathrm{ms}) \pm \sigma \\
(\mathrm{ms})\end{array}$ \\
\hline Low turb & N/A & 0.9 & 15.1 & 0.52 & $140 \rightarrow 237$ & $1.3 \rightarrow 0.8$ & $5.6 \rightarrow 1.5$ & $113 \pm 12$ \\
\hline Mid turb & Static grid & 2.8 & 7.4 & 0.54 & $213 \rightarrow 308$ & $2.0 \rightarrow 1.3$ & $0.9 \rightarrow 0.3$ & $93 \pm 14$ \\
\hline High turb & Impinging jet grid & 7.0 & 6.7 & 0.57 & $482 \rightarrow 583$ & $3.2 \rightarrow 2.3$ & $0.3 \rightarrow 0.2$ & $75.2 \pm 19$ \\
\hline
\end{tabular}

\subsection{Diagnostics}

Two PIV systems were simultaneously employed to measure the reacting flow field at two resolution levels; a wide-field, 2-component PIV system and a high-resolution, three-component (stereoscopic) system. The PIV diagnostics are synchronized with $\mathrm{C}_{2} * / \mathrm{CH}^{*}$ chemiluminescence imaging for characterizing the flame boundary [10] and optically quantifying the global equivalence ratio through time [17].

In both PIV systems, scattering is from $\mathrm{Al}_{2} \mathrm{O}_{3}$ particles (150nm nominal diameter) seeded into the flow well upstream of the bluff-body. The particles are illuminated at 532nm using the beams from a dualhead Nd:YAG laser (LDP-200MQG Dual), formed into a thin sheet using a cylindrical and spherical lens. The laser is operated at $20 \mathrm{kHz}$ repetition rate.

In the wide field, 2-component PIV system, images are acquired using a highspeed CMOS camera (Photron SA1.1). The domain of interest includes the recirculation zone and closeout region between 0.55 $\leq x / H \leq 4.75$, depicted in Fig. 1a. Images are processed in LaVision Davis software using a multi-pass 
cross correlation algorithm with a final window pass of $16 \times 16$ pixels. The final vector resolution is $\lambda_{m}=$ $950 \mu \mathrm{m}$ which is $\lambda_{m} / l_{f}=2$ relative to the laminar flame thickness of a propane-air flame at $\Phi=0.7$, and $\lambda_{m} / \lambda_{k}=14.1$ relative to the Kolmogorov length scale. The statistical correlation uncertainty in the velocity is $0.25 \mathrm{~m} / \mathrm{s}[18,19]$.

In the stereo-PIV system, images were acquired on a pair of highspeed CMOS cameras (Photron SA-Z) mounted on opposite sides of the facility. The cameras, oriented $22.5^{\circ}$ from perpendicular to the laser sheet, were equipped with schiempflug adaptors to eliminate blurring associated with off-axis imaging. The SPIV images are collected simultaneously with the wide-field PIV images. The domain for the SPIV images resides between $1.8 \leq x / H \leq 3.4$ as shown in Fig. 1a. The vector resolution for the SPIV is $\lambda_{m}=292 \mu \mathrm{m}$, which corresponds to $\lambda_{m} / l_{f}=0.89$ and $\lambda_{m} / \lambda_{k}=4.1$. The uncertainty in the velocity fields (based on correlation statistics) is $0.05 \mathrm{~m} / \mathrm{s}$.

Imaging of diatomic carbon $\left(\mathrm{C}_{2}{ }^{*}\right)$ and methylidyne $\left(\mathrm{CH}^{*}\right)$ emissions was used to characterize the duration of the blowout process $[10,13]$, to identify the instantaneous flame position, and to determine the instantaneous equivalence ratio $[17,20,21] . \mathrm{C}_{2} *$ and $\mathrm{CH}^{*}$ emissions were imaged using on a highspeed CMOS camera (Photron SA1.1) $10 \mathrm{kHz}$, where each image aligns with every other PIV/SPIV image. An image splitter and appropriate filters is used to acquire separate images of the $\mathrm{C}_{2} *$ and $\mathrm{CH}^{*}$ emission onto one sensor [17].

The process for determining the equivalence ratio from $\mathrm{C}_{2}{ }^{*}$ and $\mathrm{CH}^{*}$ intensities is documented in Reyes et al [17]. The technique involves taking the ratio of the $\mathrm{C}_{2} *$ to $\mathrm{CH}^{*}$ intensity and mapping it to an equivalence ratio. The equivalence ratio map is determined

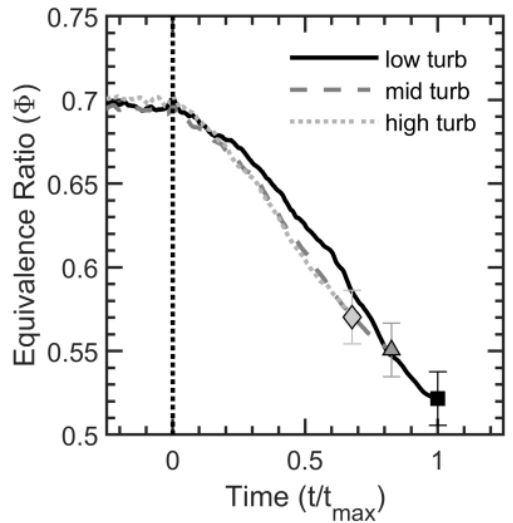

Figure 2 Temporal equivalence ratio throughout the extinction duration for all turbulence test conditions. Symbols represent the equivalence ratio at blowout. 
from calibration images which are are collected using the the same optical setup described above. For calibration, 1000 images are collected for discrete equivalence ratios between $0.5 \leq \Phi \leq 0.8$ with increments of 0.05 . This data is used to develop a curve of $\Phi$ vs $\mathrm{C}_{2} * / \mathrm{CH}^{*}$ intensity, and is used to evaluate the equivalence ratios during blowout. During blowout, the local equivalence ratio along the length of the flame is spatially averaged between $2 \leq x / H \leq 4$ for every instant in time and presented in Fig. 2. The blowout duration

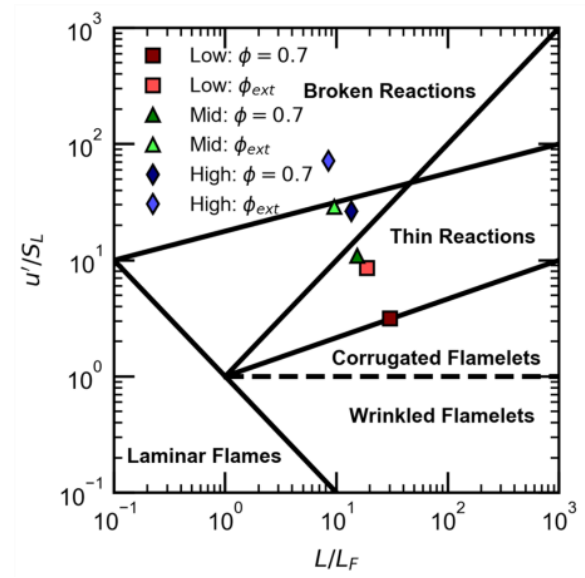

Figure 3 Combustion regime diagram for blowout test conditions.

for each test case is normalized by the blowout duration for the low turbulence case, which was $113 \mathrm{~ms}$. The blowout equivalence ratio for each test case is marked by the symbols in Fig. 2, and also included in Table 1 . The uncertainty in the equivalence ratio profiles is $\Phi \pm 0.02$ and is calculated from a propagation of uncertainty which accounts for the error in the air and fuel flow rates, the standard deviation of the $\mathrm{C}_{2} * / \mathrm{CH}^{*}$ calibration images, as well as the random error between trials. The blowout equivalence ratios were also validated using the experimental technique described by Ballal et al [5].

All test conditions are plotted on the combustion regime diagram in Fig. 3. There are two data points plotted for each condition: an initial condition for $\Phi=0.7$ and a final condition at the blowout equivalence ratio for each turbulence level. It is noteworthy that the turbulent length scales decrease with increased turbulence intensity; this is caused by the design of the turbulence generator as the impinging jets will induce small-scale turbulence. 


\section{Results and Discussion}

The results below document the evolution of the flowflame field throughout lean blowout for various freestream turbulence conditions. Specifically, the results focus on flamevorticity interactions and the local flame strain rate as a function of the freestream turbulence levels. Since local flame straining and turbulent eddies are highly dependent on time and space, the results will higlight instantaneous data obtained from a single blowout case for each turbulence condition. It is noted that the presented results and trends were consistent between repeated trials.

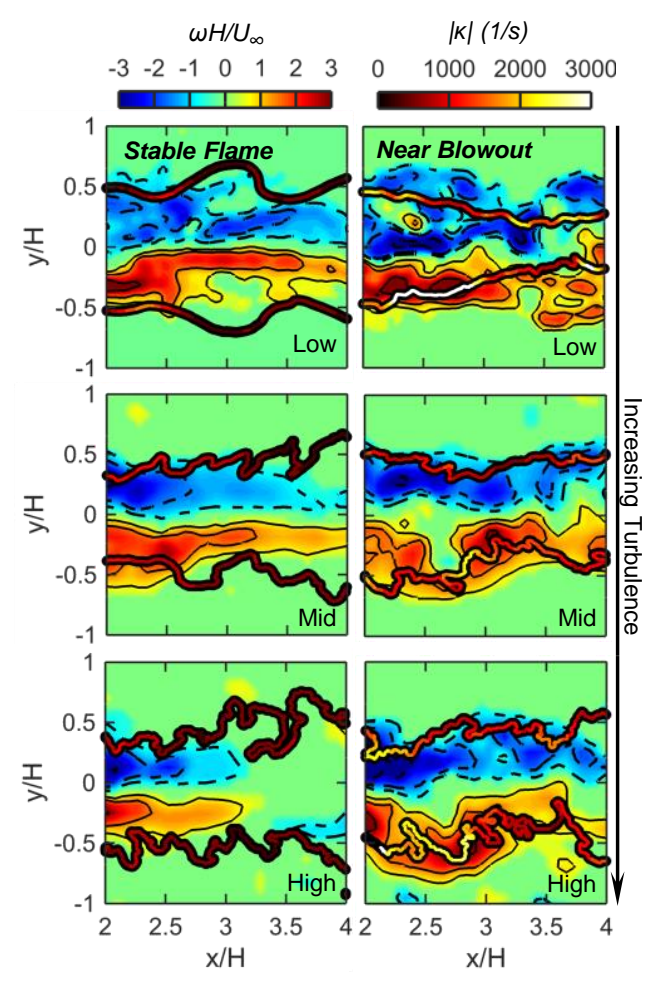

An overview of the extinguishing flame-flow field is presented in Fig. 4. The instantaneous flame boundary, vorticity

Figure 4 Instantaneous flame topology, vorticity field, and local flame strain rate for all turbulent test cases.

fields, and local flame strain rates are depicted for two distinct time instances: one instant represents a stable flame where $\Phi=0.7$ and the other depicts a flame near blowout. The instantaneous flame boundary is determined from the Mie scatter images using a statistical threshold to identify the seed-density jump from the flames heat release [7,22]. A median filter (10x10 window size) is first applied to the images to eliminate noise. The image intesnity is then inverted to provide a higher contrast between the reactant and product regions. Finally a statistical threshold is calculated using Otsu's method, and the flame boundary is extracted using this threshold [10]. The flame boundary was also validated using the $\mathrm{C}_{2}{ }^{*} / \mathrm{CH}^{*}$ images; the root mean square deviation between the two techniques was less than $1.6 \mathrm{~mm}$ throughout the blowout 
duration. It is also noted that the flame boundary in Fig. 4 is exaggerated to highlight the local strain rate along the flame edge. The local strain rate along the flame is calculated using eq. (1) $[7,8,12,23]$.

$\kappa=-n_{x} n_{y}(\partial u / \partial y+\partial v / \partial x)+\left(1-n_{x}^{2}\right) \partial u / \partial x+\left(1-n_{y}^{2}\right) \partial v / \partial y$

In eq. (1), $u$ and $v$ are the streamwise and cross-stream velocity components and $n_{x}$ and $n_{y}$ are the local horizontal and vertical normal components along the flame boundary.

For the stable flames in Fig. 4, the flame boundaries extend beyond the shear layer vorticity regions and the strain rate along the flame is low. Near blowout, the flame boundary resides closer to the shear layer vorticity regions due to the reduced flame speed $[7,8]$, which causes the flame boundary to overlap with the shear layer vorticity (Fig. 1) and results high-magnitude strain rates on

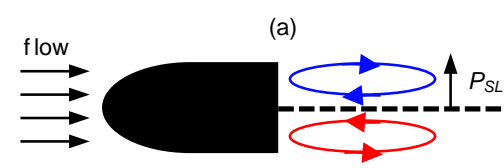

(b)
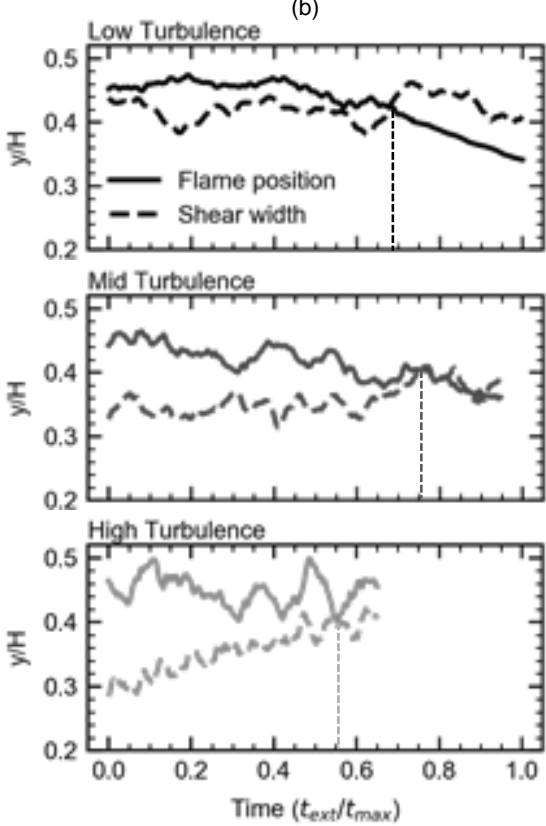

Figure 5 (a) Sketch of vertical shear layer position and (b) mean transverse flame position and shear layer width through time (both quantities spatially averaged from $2 \leq x / H \leq 4)$. the flame.

To compare the flame and shear layer interactions between the three turbulence conditions, the mean shear layer position and the vertical flame position are evaluated and shown in Fig. 5. The vertical shear layer position is calculated as the vertical distance from the bluff-body centerline to the point in the freestream where the vorticiy reaches zero. A visual representation is provided in Fig. 5a and the calculated values are shown in Fig. 5b. The mean transverse flame position is also provided in Fig. 5b. For each turbulence case, there is a general convergence between the locations of the shear layer and the flame. The time instant where the flame position and shear layer first overlap is also marked for each turbulence 
condition in Fig. 5b. For the low turbulence case, after the initial flame and shear layer overlap, the flame edge remains inside the shear layer region. As the turbulence increases, the interaction between the flame and shear layer diminishes. For instance, the high turbulence case experiences a single instant where the flame position and shear layer overlap. Beyond this time instant, the mean flame position remains outside of the shear layer vorticity. This indicates that the interaction between the flame and the shear layer is diminished with increased turbulence. The reduced interaction is a result of the higher turbulent flame speed as presented in Table 1. The effect of the turbulent flame speed can also be visualized in Fig. $5 \mathrm{~b}$ when differentiating the mean transverse flame positions at blowout. For the low, mid, and high turbulence conditions, the flame positions at blowout are $y / H=3.4,3.6$, and 4.6, respectively. It should also be noted that the shear layer vorticity and recirculation zone length both decrease with turbulence [12], meaning that flame
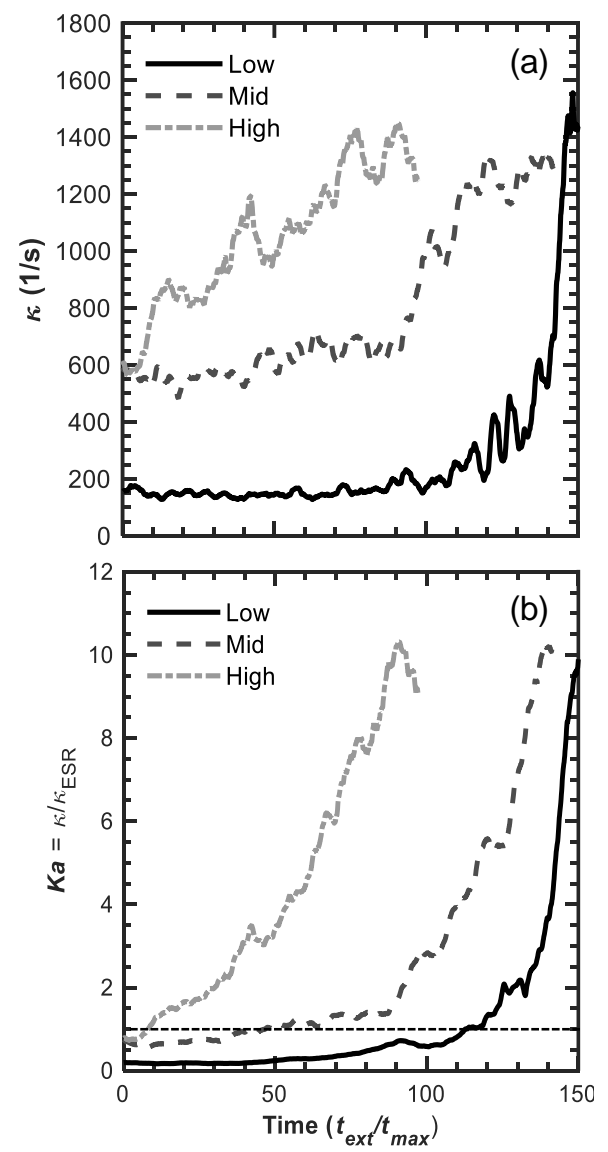

Figure 6 (a) Strain rate along the flame, spatially averaged between $2 \leq \mathrm{x} / \mathrm{H} \leq 4$, throughout the extinction duration. (b) Karlovitz number along the flame throughout extinction.

and shear layer interactions will be mitigated at high turbulence conditions $[8,12]$.

The strain rate along the flame for all turbulence conditions is presented in Fig. 6a. For all cases, the strain rate increases through time. However, the highest turbulence case experiences the largest strain rate magnitude throughout its lifetime. Additionally, a Karlovtiz number is provided in Fig. 6b. The Karlovtiz number is calculated as the ratio of the flame strain rate $(\kappa)$ to the extinction strain rate $\left(\kappa_{E S R}\right)$. The $\kappa_{E S R}$ values are calculated using chemkin-opdiff for premixed opposed jet propane-air flames. Using 
the equivalence ratio data from Fig. 2 , it is possible to determine $\kappa_{E S R}$ as a funciton of time. The strain rate curves are then divided by the $\kappa_{E S R}$ curves to obtain the $K a$ data in Fig. 6 b. Based on this formulation, $K a$ $\geq 1$ is where localized extincitons should occur along the flame. Increased $K a$ values will be coupled with flame stretching [2,7-9,11], meaning that localized extincitons are more likely to occur as the turbulence levels are increased. However, flame stretching is also a function of flame curvature [2,9]. Therefore, the local flame curvature along the flame is evaluated and presented as a probability density function (PDF) in Fig. 7. To obtain the PDFs, the local curvature is

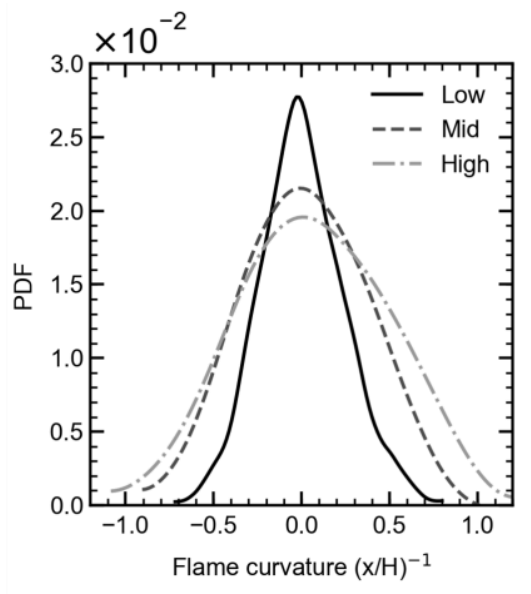
calculated using eq. (2) along the flame coordinates between $2 \leq x / H$

Figure 7 PDF of flame curvature for all turbulent test cases. $\leq 4$ for every instant in time.

$$
C=\left(x^{\prime} y^{\prime \prime}-y^{\prime} x^{\prime \prime}\right) /\left(x^{\prime 2}+y^{\prime 2}\right)^{3 / 2} \equiv 1 / r
$$

The PDFs reveal that increasing turbulence leads to larger flame curvature values. The combination of increased curvature and strain rates verify that the flame is exposed to greater stretching effects with increased turbulence.

The high-resolution SPIV is used to visualize fine-scale interactions between the flame and flow field. The flame boundary, vorticity field, and local flame strain rates are evaluated from the SPIV images and presented in Fig. 8. Similar to Fig. 4, one time instant depicts a stable flame (left) and another instant shows the flame-flow field near blowout (right). As the turbulence increases,

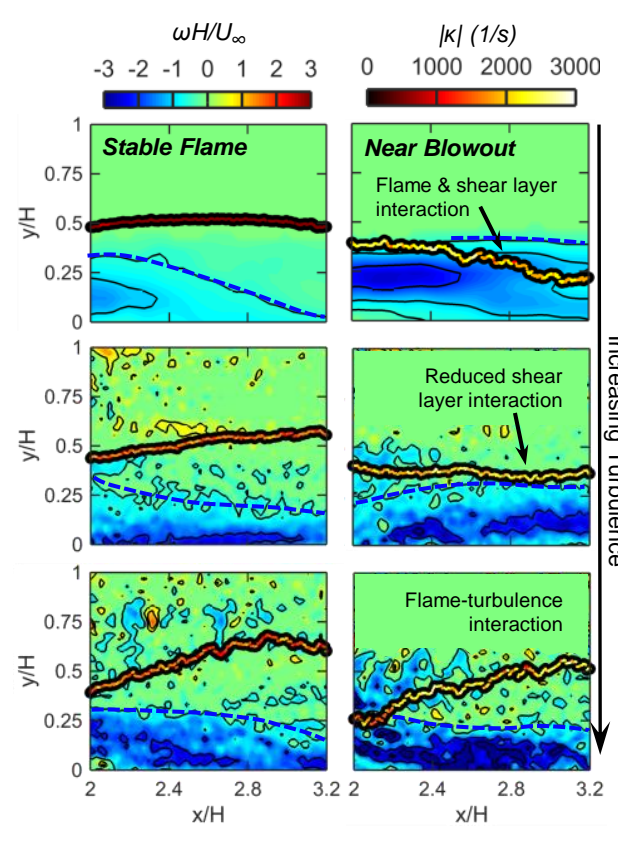

Figure 8 Instantaneous flame boundary, vorticity field*, and flame strain rate obtained from the stereo PIV. The dashed blue line represents the boundary of the shear layer. $*$ A portion of the frestream vorticity has been removed in some frames for legible annotations. 
small-scale eddy structures become visible in the freestream regions of Fig. 8. The quantity and magnitude of the eddy structures increase with turbulence.

The low turbulence condition shows a clear overlap between the flame boundary and the shear layer vorticity, while the high turbulence

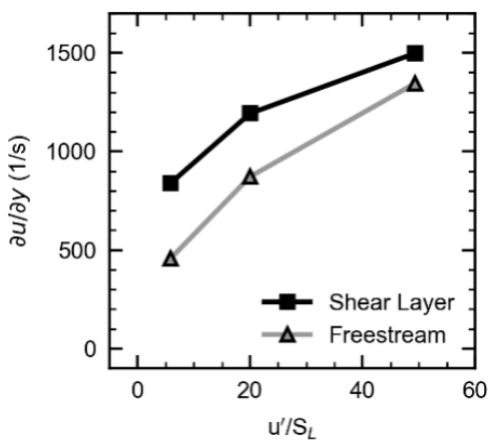
case shows no clear interaction between the two. Instead, the highturbulence flame boundary resides in the freestream, where it overlaps

Figure 9 Comparison of the $\partial u / \partial y$ velocity gradient between the freestream and the shear layer. with the strong turbulent eddies. The flame-eddy overlap subjects the flame to freestream velocity gradients that induce high strain rates along the flame that match the same order of magnitude as the shear layer. To verify this, the velocity gradients within the shear layer are compared to the velocity gradients within the freestream and presented within Fig. 9. Since the global shift in the flame position is in the transverse (y) direction, and the bulk flow direction is in the streamwise (x) direction, the primary velocity gradient of interest is the $\partial u / \partial y$ gradient; this is the dominant velocity gradient that will impose highmagnitude strain along the flame as the flame speed decreases. The $\partial u / \partial y$ velocity gradient is calculated across the thickness of the shear layer and in the freestream portions of the domain. The spatial and temporal mean value of the two $\partial u / \partial y$ gradients are evaluated in Fig. 9. As the freestream turbulence increases, the $\partial u / \partial y$ velocity gradient approaches the same magnitude as the shear layer gradient.

These obervations indicate that the extinction and blowout processes are highly dependent on the freestream turbulence. As the equivalence ratio decreases, the converging positions of the flame boundary and the shear layer vorticity expose the flame to decreased fluid time scales and elongated chemical timescales. When the ratio of these two timescales (the Damköhler number, $D a=\tau_{\text {flow }} / \tau_{\text {chem }}$ ), becomes too small, local extincitons initiate. However, the presence highly turbulent eddies within the freestream (Fig. 
8) cause the local fluid timescales along the flame to decrease, which in turn decrease the local $D a$ as documented in Table 1. For the mid and high turbulence cases, the initial $D a$ is less than unity for the stable condition of $\Phi=0.7$, which suggests that localized flame extinciton can occur within the freestream prior to interacting with the shear layer. The increased probability of local extinciton will augment the overall blowout process as demonstrated by the increased strain rate along the flame (Fig. 6) as well as the shorter lifespan and increased blowout equivalence ratio for the high turbulence condition (Table 1). Consequently, flame-turbulence intractions expedite the extincition and blowout processes, in a manner similar to those described for for a turbulent jet flames [24] or turbulent diffusion flames [25]. In both configurations, the turbulence induces localized velocity gradients which promote localized flame straining and lead to blowout.

\section{Conclusions}

This research characterized the temporal blowout process for bluff-body flames subjected to varying turbulence conditions. For all test cases, the positions of the flame and shear layer converged as the equivalence ratio was reduced, causing the strain rate to increase through time. However, the high turbulent flame maintained the largest strain rate throughout its lifetime, causing it to blowout the fastest. Under further investigation, the SPIV data revealed that the turbulent flame overlaps with freestream turbulent eddies, invoking a high-magnitude strain rate along the flame prior to interacting with the shear layer. This was verified by comparing the $\partial u / \partial y$ velocity gradient between the freestream and the shear layer. In this manner, flame-turbulence interactions increase the likelihood of local extinction to occur, which will accelerate the overall blowout process. 


\section{Acknowledgments}

The work is sponsored by the Air Force Office of Scientific Research award numbers 19RT0258/FA9550-19-0322 and FA9550-16-1-0044; Program Manager: Dr. Chiping Li. The authors would like to acknowledge Dr. Campbell Carter at the AFRL for providing equipment support for performing these experiments.

\section{References}

[1] D. Dunn-Rankin, Lean Combustion: technology and control, Academic Press, Burlington, MA, 2008.

[2] S.J. Shanbhogue, S. Husain, T. Lieuwen, Lean Blowoff of Bluff Body Stabilized Flames: Scaling and Dynamics, Prog. Energy Combust. Sci. 35 (2009) 98-120.

[3] M.V. Herbert, Aerodynamic influences on flame stability, Prog. Combust. Sci. Technol. (1960) 61109.

[4] R.I. Ozawa, Survey of basic data on flame stabilization and propagation for high speed combustion systems, OH, Technical Report TR-70-81, 1971.

[5] D. Ballal, A.H. Lefebvre, Weak extinction limits of turbulent flowing mixtures, J. Eng. Power. 101 (1979) 343-348.

[6] A.H. Lefebvre, D.R. Ballal, Gas Turbine Combustion, CRC Press, 2010.

[7] S.G. Tuttle, S. Chaudhuri, K.M. Kopp-Vaughan, T.R. Jensen, Lean blowoff behavior of assymetrically-fueled bluff body-stabilized flames, Combust. Flame. 160 (2013) 1677-1692.

[8] S. Chaudhuri, S. Kostka, M.W. Renfro, B.M. Cetegen, Blowoff dynamics of bluff body stabilized turbulent premixed flames, Combust. Flame. 157 (2010) 790-802.

[9] S. Nair, T. Lieuwin, Near-Blowoff Dynamics of a Bluff-Body Stabilized Flame, J. Propuls. Power. 23 (2007) 421-427.

[10] A.J. Morales, I.M. Lasky, M.K. Geikie, C.A. Engelmann, K.A. Ahmed, Mechanisms of Flame Extinction and Lean Blowout of Bluff Body Stabilized Flames, Combust. Flame. 203 (2019) 3145.

[11] S.G. Tuttle, S. Chaudhuri, S. Kostka, K.M. Kopp-Vaughan, T.R. Jensen, B.M. Cetegen, M.W. Renfro, Time-resolved blowoff transition measurements for two-dimensional bluff body-stabilized flames in vitiated flow, Combust. Flame. 159 (2012) 291-305.

[12] B.R. Chowdhury, B.M. Cetegen, Effects of free stream flow turbulence on blowoff characteristics of bluff-body stabilized premixed flames., Combust. Flame. 190 (2018) 302-316.

[13] J. Kariuki, J.R. Dawson, E. Mastorakos, Measurements in turbulent premixed bluff body flames close to blow-off, Combust. Flame. 159 (2012) 2589-2607.

[14] C.M. Vagelopoulos, F.N. Egolfopouols, C.K. Law, Further considerations on the determination of laminar flame speeds with the counterflow twin-flame technique, Proc. Combust. Inst. 25 (1994) 1341-1347.

[15] N. Peters, Turbulent Combustion, Cambridge University Press, U.K., 2000. 
[16] N. Peters, The turbulent burning velocity for large-scale and small-scale turbulence, J. Fluid Mech. 384 (1999) 107-132.

[17] J. Reyes, R.K.A. Kailasanathan, Relationship between the chemiluminescence intensity rato of C2* and $\mathrm{CH}^{*}$, charge pressure, and gasoline, Energy and Fuels. 32 (2018) 10933-10940.

[18] Z. Carr, K. Ahmed, D. Forliti, Spatially correlated precision error in digital particle image velocimetry measurements of turbulent flows, Exp. Fluids. 47 (2009) 95-106.

[19] A. Sciacchitano, B. Wieneke, PIV uncertainty propagation, Meas. Sci. Technol. 27 (2016) 1-16.

[20] T.P. Clark, Studies of $\mathrm{OH}, \mathrm{CH}$, and $\mathrm{C} 2$ radiation from laminar and turbulent propane-air and ethylene-air flames, Cleveland, OH, 1958.

[21] Y.K. Jeong, C.H. Jeon, Y.J. Chang, Evaluation of the equivalence ratio of the reacting mixture using intensity ratio of chemiluminescence in laminar particlly premixed CH4-air flames, Exp. Therm. Fluid Sci. 30 (2006) 663-673.

[22] S. Pfadler, F. Beyrau, A. Leipertz, Flame front detection and characterization using conditioned particle image velocimetry, Opt. Express 15 (2007) 15444-15456.

[23] S.H. Chung, C.K. Law, An invariant derivation of flame stretch, Combust. Flame. 55 (1984) 123125.

[24] T.F. Guiberti, W.R. Boyette, A.R. Marsi, W.L. Roberts, Detachment mechanisms of turbulent nonpremixed jet flames at atmospheric and elevated pressures, Combust. Flame. 202 (2019) 219-227.

[25] A. Yoshida, T. Igarashi, Y. Kotani, Extinction of turbulent diffusion flames by Kolmogorov microscale turbulence, Combust. Flame. 109 (1997) 669-681. 